\title{
Colaboración entre Docentes y Directivos: Estudio de Caso del Desarrollo Profesional e Inclusión en Pandemia
}

\author{
Collaboration between Teachers and Management Team: A \\ Case Study on Professional Development and Inclusion in \\ Pandemic
}

\author{
Juan Pablo Queupil Quilamán * \\ Catalina Cuenca Vivanco \\ César Maldonado Díaz
}

Universidad Católica Silva Henríquez, Chile

\begin{abstract}
La colaboración en educación ha sido considerada relevante para el desarrollo profesional de los actores escolares. No obstante, poco se sabe de esto en contextos de pandemia por Covid-19, que han exacerbado las inequidades socioeducativas y reconfigurado los patrones de colaboración, así como el fomento o inhibición de nuevos aspectos y habilidades en docentes y directivos escolares. A través de un estudio de caso de una escuela rural en Chile, con un enfoque secuencial mixto, este estudio muestra que la colaboración ha aumentado durante la emergencia sanitaria, junto con la preponderancia de los integrantes que abordan la inclusión educativa. Las dimensiones contextuales y en diversos niveles condicionan esta colaboración y desarrollo profesional, tales como la reciente nueva administración la educación pública que sobredemanda a los actores de las escuelas, las destrezas asociadas con la educación remota, así como la necesidad de reforzar las habilidades socioemocionales del cuerpo docente y directivo en pandemia. Esto conlleva el potencial de una mejor articulación entre los distintos niveles del sistema educativo y en la propia escuela que considere el desarrollo profesional contextualizado de manera pertinente y atingente, en particular en un escenario post-pandemia $y$ otras potenciales emergencias coyunturales en educación.
\end{abstract}

Descriptores: Cooperación; Personal docente; Personal directivo; Desarrollo profesional; Estudio de caso.

Educational collaboration has been considered a relevant aspect for the professional development of school staff. However, little is known about this in the context of the Covid-19 pandemic, which has intensified socio-educational inequalities and reconfigured collaboration patterns, as well as the promotion or inhibition of new facets and skills among teachers and school administrators. Through a case study of a rural school in Chile, based on a mixed sequential approach, this study shows that collaboration has increased due to the Covid emergency, along with the preponderance of school members who address educational inclusion. Contextual dimensions at different levels affect this collaboration and professional development, such as the recent new administration of public education that has overpressured school actors, the skills associated with on-line education, as well as the need to reinforce socio-emotional abilities of teaching and management staff within a pandemic scenario. This entails the potential for a better articulation between the different levels of the educational system and in the school, that considers contextualized professional development in a pertinent and appropriate way, particularly in a post-pandemic scenario and other potential conjunctural emergencies for educational settings.

Keywords: Cooperation; Teaching personnel; Managerial staff; Career development; Case study. 


\section{Introducción}

Los procesos de mejora escolar en Chile relacionados con la inclusión escolar han promovido la colaboración como uno de los elementos relevantes del desarrollo profesional de quienes integran las comunidades escolares (Ávalos-Bevan y Bascopé, 2017). Esto ha llevado a que el desarrollo profesional se estudie en diversas investigaciones y se fomente como una actividad fundamental (Ávalos, 2013; UNESCOOREALC, 2013; Vaillant y Marcelo, 2015), donde miembros de establecimientos escolares comparten experiencias, analizan y examinan colectivamente sus prácticas pedagógicas (MINEDUC, 2019; Vaillant, 2016). Esto explica lo relevante de las relaciones colaborativas entre docentes y directivos en las escuelas como campo de estudio.

Además, en el contexto chileno destaca la creación de los Programas de Integración Escolar (PIE) como un marco para la integración escolar que modifica la normativa pública que regula la inclusión escolar. Una de las propuestas para el funcionamiento del PIE es la práctica del trabajo colaborativo en equipos multidisciplinarios al interior de las escuelas adscritas al programa. Los equipos de aula pueden estar compuestos por docentes, educadores(as) diferenciales-especiales, fonoaudiólogos(as) y psicólogos(as), con el fin de enseñar a todos los estudiantes en aulas escolares. Junto con esto, se establece la modalidad de trabajo compartido entre docentes, conocida como co-enseñanza (MINEDUC, 2009, 2017).

En este escenario, la pandemia por Covid-19 ha tensionado a las comunidades escolares en diversas dimensiones (Gajardo-Asbún et al., 202 1; Lizana et al., 2021; Ramos-Huenteo et al., 2020; Salas et al., 2020). Es por esto que el presente estudio investigó cómo las prácticas de colaboración fueron afectadas en este contexto y cómo los actores antes mencionados se relacionan entre sí en miras de su desarrollo profesional.

En ese sentido, este artículo de investigación tiene por objetivo examinar las prácticas colaborativas en los procesos de enseñanza de diversos actores escolares de una comunidad escolar, junto con detectar el rol que estos actores juegan en estas interacciones tanto antes como durante la pandemia, para responder a la siguiente pregunta: ¿Cuáles son las prácticas, roles, patrones, desafíos y oportunidades en la colaboración que se establecen entre docentes, directivos y miembros del Programa de Integración Escolar y su relación con el desarrollo profesional durante la pandemia Covid19? A través de esta pregunta, el artículo espera ser un aporte al conocimiento sobre prácticas colaborativas entre docentes, directivos escolares y miembros del PIE que impactan en el desarrollo profesional en el marco de la inclusión escolar en contextos complejos como la pandemia.

\section{Colaboración para el desarrollo profesional docente}

Las discusiones teóricas sobre colaboración y el cómo este tipo de relaciones inciden en el desarrollo profesional docente se han centrado principalmente en aspectos como la cantidad de docentes necesaria para sostener este tipo de trabajo, la diferencia con la autonomía docente, o la ausencia de definiciones epistemológicas sobre este constructo teórico (Vangrieken et al., 2015, 2017). En este contexto, la colaboración se entiende como

las mediaciones o procesos estructurados o semiestructurados (como asociaciones, redes de colaboración) o contextos informales (como las interacciones en el lugar de trabajo) que faciliten el aprendizaje y estimulan a 
los profesores a alterar o reforzar las prácticas docentes y educativas. (Ávalos, 2011, p.12)

Respecto a las condiciones y factores para el desarrollo profesional en el marco de la colaboración, se han planteado dos tipos de condiciones distintas. En primer lugar, las de nivel macro, que incluyen el funcionamiento de los sistemas educativos, las reformas políticas y las condiciones de trabajo de los profesores, además de los factores históricos que determinan lo que se acepta o no como formas adecuadas para el desarrollo profesional (Coskie y Place, 2008; Skerrett, 2010). En segundo lugar, aquellas que remiten a las comunidades escolares y sus territorios, abarcando el funcionamiento de las estructuras administrativas y orgánicas, cómo éstas interactúan para facilitar o restringir el aprendizaje de los profesionales en el lugar de trabajo, los elementos que componen las escuelas en diferentes ubicaciones geográficas, y las oportunidades para el aprendizaje de los profesionales. Este nivel ilustra cómo las creencias, tradiciones y tipos de decisiones institucionales afectan el grado de participación informal y formal de los docentes en los intercambios pedagógicos (Jurasaite-Harbison y Rex, 2010; Sato y Kleinsasser, 2004). El siguiente estudio se enmarca en esto último, ya que examina la relación entre factores que facilitan o complejizan la concreción de relaciones colaborativas, principalmente en el intercambio de prácticas, nivel de participación y la preparación de profesionales (GarcíaMartínez et al., 2021), pues en Chile se ha hecho evidente la necesidad de abordar la colaboración con miras al desarrollo profesional docente desde una perspectiva integral, que considere las características contextuales o territoriales dentro de la formación continua de los docentes y la evaluación de su labor. Esto permitiría abordar las características situadas de las comunidades y las interacciones colaborativas que se desarrollan en ellas (Aparicio-Molina y Sepúlveda-López, 2019).

\section{Prácticas colaborativas}

En Chile existen diferentes percepciones entre docentes y directivos escolares sobre cómo implementar las prácticas colaborativas, principalmente por la comprensión diversa sobre esta, ya que no cuenta con una mirada ni estimación única. Estas visiones consideran una serie de factores, entre los que destacan las características de la organización escolar y su gestión, la cultura escolar de los establecimientos, la formación inicial y el perfeccionamiento docente recibido, principalmente en torno a la diversidad y concepciones del aprendizaje, que se traducen en una determinada práctica pedagógica (Tenorio, 2005). Los estudios sobre el tema en comunidades escolares donde se instalan los PIE han discutido las barreras para las prácticas conjuntas de enseñanza (coenseñanza) y las concepciones docentes sobre la integración/inclusión escolar. Sin embargo, en la actualidad, esto se constituye como responsabilidad de las comunidades escolares en su totalidad y no sólo de los PIE. Además, progresivamente se han instalado las Comunidades Profesionales de Aprendizaje (CPA) como espacios que promueven la colaboración entre colegios en territorios escolares, así como también dentro de comunidades escolares particulares.

\subsection{La co-enseñanza como práctica colaborativa}

La investigación chilena sobre co-enseñanza, a diferencia de la internacional, es de corto alcance y reciente desarrollo, centrándose en las experiencias conjuntas entre docentes al interior de escuelas municipalizadas y sus dificultades en la implementación de la política pública. 
Existen dos grupos de estudios sobre co-enseñanza. En primer lugar, aquellas que indagan sobre las concepciones de los profesores que co-enseñan, y que muestran las diferencias que persisten en las concepciones entre profesores generalistas y diferenciales y los diversos perfiles de relación que se establecen desde ellas, las cuales son conflictivas entre sí (López et al., 2014; Muñoz et al., 2015). Además, los estudios sobre la forma de desarrollo de la co-enseñanza muestran el bajo grado de colaboración entre los docentes, apreciándose la influencia de concepciones tradicionales y discriminadoras entre las modalidades de educación regular y educación común, donde la práctica predominante en la co-enseñanza es el apoyo docente (Rodríguez, 2012a), mostrando también una serie de facilitadores y barreras en esta práctica que requiere un fuerte apoyo institucional para su desarrollo (Figueroa-Céspedes et al., 2020; Urbina et al., 2017).

\subsection{Barreras en la co-enseñanza}

Rodríguez Rojas (2012) y Rodríguez Rojas y Ossa (2014) desarrollaron estudios exploratorios cualitativos con el propósito de develar la percepción sobre el trabajo colaborativo que tienen los profesores de educación regular y especial, en el contexto del PIE, señalando el bajo grado de colaboración entre los docentes, además de la influencia de concepciones tradicionales y discriminadoras entre la educación regular y especial. En la misma línea de experiencias en aulas escolares, Urbina y otros (2017) encuentran dos elementos relevantes. En primer lugar, que la práctica predominante en la co-enseñanza de parejas de docentes se relaciona con el modelo de apoyo en que uno de los dos tiene mayor protagonismo en clases. En segundo lugar, señalan elementos facilitadores (jornadas laborales similares, desarrollo de la confianza profesional) y barreras para las prácticas de co-enseñanza (ausencia de tiempo de trabajo, descoordinación, falta de apoyo directivo). Por su parte, Navarro-Aburto y otros (2016) encuentran que el profesorado y personal encargado del PIE cumplen con normativas y requerimientos del Decreto $\mathrm{N}^{\circ}$ 170, en relación con las adaptaciones curriculares, pero está sujeto a la informalidad en la articulación y planificación debido a la falta de tiempo y recursos disponibles. De este modo, plantean que existe una necesidad constante de adaptaciones curriculares, pues son un mecanismo que permite lograr la individualización de la enseñanza, mejorando la práctica colaborativa entre docentes.

\subsection{Las CPA como práctica colaborativa}

Las CPA son un modelo de prácticas colaborativas que progresivamente se han instalado en Chile (Venegas et al., 2010), que permite abordar la tensión sobre el trabajo docente, la mejora de las prácticas pedagógicas y los resultados de aprendizaje del estudiantado (Bolívar y Bolívar, 2013), basándose en la mirada compleja de las instituciones escolares, ya que estas cambian y en cierta manera evolucionan frente a las situaciones asociadas a la enseñanza, el aprendizaje de los estudiantes y el trabajo docente colaborativo (Rodríguez-Gómez y Gairín-Sallán, 2015). Por lo tanto, las CPA serían una respuesta concreta a los cambios desarrollados en los establecimientos escolares.

Lo anterior permite la configuración de una serie de elementos que promueven la colaboración: desarrollo de espacios de participación, interacción sobre los resultados del estudiantado, posibilidad de cambio en la cultura escolar a largo plazo, sistematicidad en la participación y el impacto en el cambio epistemológico sobre la colaboración (Guerra et al., 2020; Philpott y Oates, 2017). Para promover la instalación de las CPA, se necesita la existencia de una relación directa y significativa con la disposición del tiempo y la regularidad en los espacios de trabajo, lo que permitiría facilitar el trabajo colaborativo y 
el encuentro en pos de la reflexión pedagógica (Cochran-Smith et al., 2015; Hairon y Tan, 2017).

\section{Método}

El siguiente artículo se enmarca en un estudio de caso (Yin, 2003) bajo un enfoque de métodos mixtos secuencial exploratorio (Creswell y Plano Clark, 2017), primeramente utilizando teorías y herramientas cuantitativas del Análisis de Redes Sociales (ARS) (Wasserman y Faust, 1994) para examinar las relaciones colaborativas que emergen en contexto de pandemia en una escuela rural de Chile, al alero administrativo del Estado a través de uno de los nuevos Servicios Locales de Educación Pública ${ }^{1}$ (Ley ${ }^{\circ}$ 21.040, 2017).

Para ello, se aplica un cuestionario sociométrico (Scott, 2000) a profesionales de la educación de la comunidad escolar, todos ellos involucrados en los procesos de mejora colaborativa, sobrepasando el $80 \%$ de respuestas deseable para realizar el análisis de redes (Moolenaar, 2012). Específicamente, este cuestionario identifica a qué profesionales de la educación recurrieron previo y durante la pandemia (es decir, con quienes interactuaron), utilizando una escala Likert de frecuencia, que va desde $0=$ Nunca hasta $4=$ Casi a diario.

Luego, se recurre al mapa de red o sociograma de la escuela (Scott, 2000) para graficar las relaciones colaborativas, distinguiendo con diferentes formas geométricas a directivos, docentes y equipo PIE, y donde las flechas entre actores representan la colaboración presente.

En segundo lugar, se profundiza en lo anterior utilizando un enfoque cualitativo basado en grupos focales (Stewart y Shamdasani, 2014) con integrantes de la escuela. En estos grupos focales indagan tres dimensiones sobre la colaboración de la escuela en pandemia: (a) prácticas, (b) roles y (c) desafíos, beneficios y proyecciones. El análisis descriptivo temático (Peel, 2020) de las transcripciones de los grupos focales fue realizado por los autores del estudio, triangulando la codificación e identificación los aspectos encontrados (Jonsen y Jehn, 2009), y así distinguir las similitudes y contrastes entre los actores de la escuela en cada dimensión analizada. Todos los participantes del estudio firmaron consentimientos informados aprobados por el comité de ética de la universidad para garantizar la anonimidad y confidencialidad de los datos.

El cuestionario sociométrico fue respondido por 26 de los 30 profesionales de la educación de la comunidad escolar donde se realizó el estudio, entre ellos directivos, docentes e integrantes del PIE (educadores diferenciales y apoyos pedagógicos). De estos, 12 actores fueron invitados a participar de los 3 grupos focales, separándolos de acuerdo con los fines del estudio, vale decir: 4 directivos (Dirección, Jefatura de Unidad Técnica Pedagógica, Inspectoría General y Coordinadora de Convivencia), 4 docentes (de diversas espacialidades), 4 integrantes del equipo PIE (Coordinación y 3 miembros más), salvaguardando la diversidad en cada grupo al considerar los indicadores de ARS de la primera etapa.

${ }^{1}$ Ley $\mathrm{N}^{\circ} 21.040$ (2017). Crea el Sistema de Educación Pública. Diario Oficial de Chile, 24 de noviembre de 2017. 


\section{Resultados}

\subsection{Red colaborativa en pandemia}

La primera parte del estudio consiste en un análisis de las interacciones que ocurrieron en la escuela durante los años 2019 y 2020. La figura 1 incluye la frecuencia de interacción con la cual directivos (triángulos), docentes (cuadrados) e integrantes del PIE (diamantes) recurrieron a sus colegas, desarrollando colaboración durante el primer semestre escolar del año 2020 (durante la pandemia). El tamaño de las figuras es más grande a mayor cantidad de menciones, así como el grosor de las flechas aumenta con la frecuencia de interacción declarada.

\section{Figura 1}

\section{Sociograma colaborativo durante la pandemia}

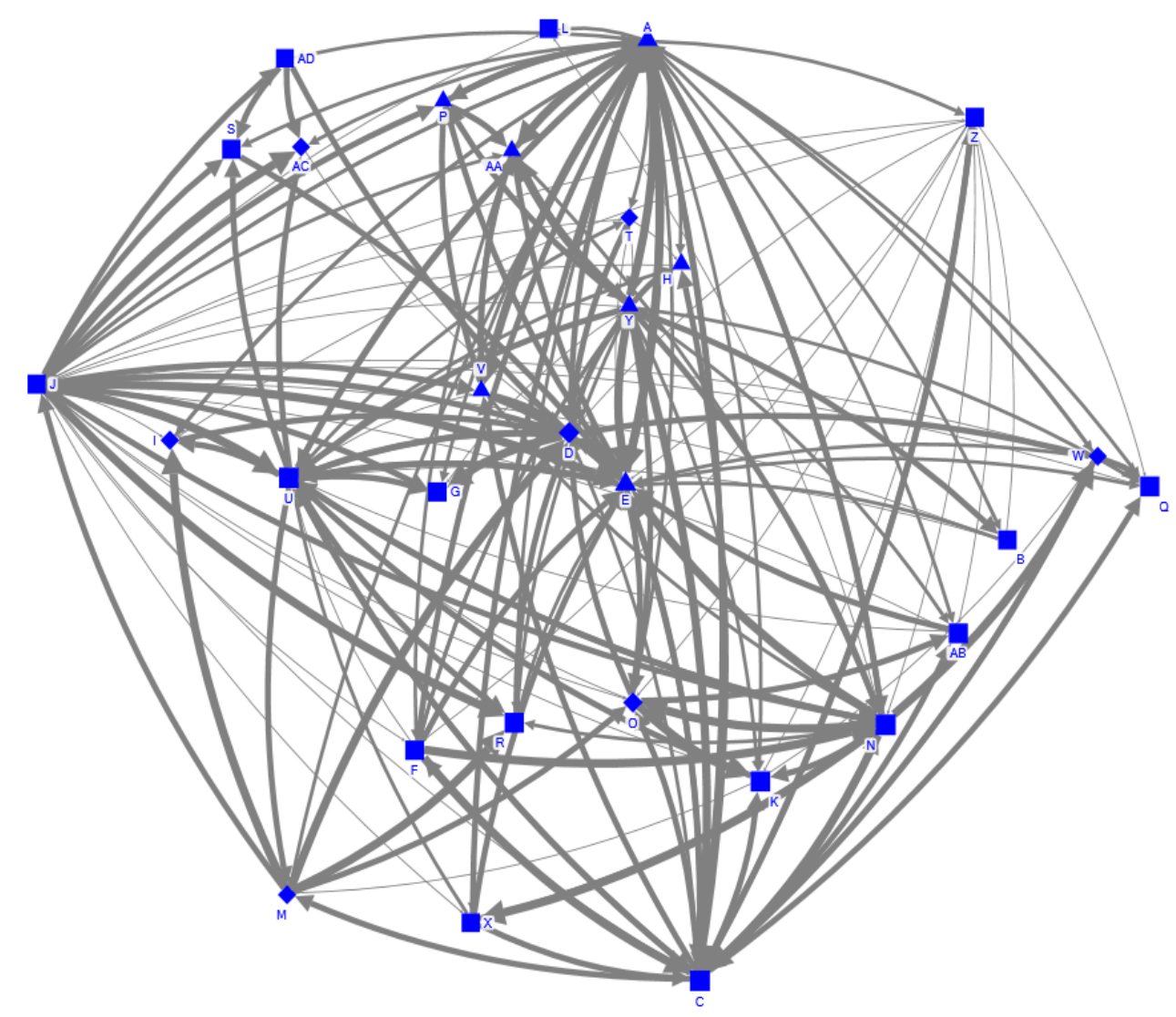

$\mathrm{El}$ análisis de los indicadores y de los sociogramas establecen que durante la pandemia se ha incrementado la frecuencia de interacciones entre los miembros del establecimiento y los integrantes del equipo del PIE, lo cual podría estar relacionado a la necesidad de desarrollar nuevas estrategias de enseñanza en el establecimiento que permitan responder a los desafíos por la emergencia sociosanitaria, posicionando los procesos colaborativos como una forma de promover el desarrollo profesional como parte de la práctica docente cotidiana, lo cual se profundiza en la siguiente sección mediante los grupos focales realizados.

\subsection{Prácticas colaborativas para la enseñanza-aprendizaje de estudiantes}

Una de las subdimensiones detectadas sobre prácticas colaborativas se relaciona con las prácticas para la enseñanza-aprendizaje de los estudiantes: cómo docentes e integrantes 
PIE trabajan conjuntamente en intervenciones dentro y fuera del aula. Una forma de colaboración considera la planificación conjunta de trabajo personalizado con estudiantes con necesidades educativas especiales y con estudiantes que requerían nivelar conocimientos desfasados:

Se hace una intervención a nivel de un aula de recurso que es virtual igual, y que es para reforzar cosas que no han podido lograr. Por ejemplo ... que están retrasados en él, aprender a leer. (Integrante PIE 2)

Así, el trabajo de co-docencia se entiende como trabajo colaborativo para la planificación y realización de clases, pero también como el trabajo personalizado que realizan los integrantes del PIE con los estudiantes. Varios entrevistados señalan que el rol principal del programa es el apoyo al profesorado de asignatura. Durante la pandemia, esto incluyó el monitoreo de aprendizaje de los estudiantes:

Mira, teníamos reuniones para organizar las clases. Entonces, los temas que íbamos a utilizar, las partes que cada uno nos correspondía. Si teníamos alguna dificultad también la que tenía mayor habilidad nos apoyaba y teníamos reuniones, yo creo que eran semanales. (Docente 3 )

\subsection{Prácticas colaborativas para la reflexión docente}

Otra práctica incluye los espacios de reflexión, donde los participantes discuten y comparten experiencias de su trabajo. Además, algunos docentes mencionan comunidades de aprendizaje para fomentar la reflexión y miembros del equipo PIE expresan que existe una falta de tiempo que dificulta su participación en estos espacios:

Entonces para eso yo creo que ahora, con esta implementación de las comunidades de aprendizaje, no sólo por ciclos, sino que por afinidad ... en el fondo que en algunas veces se nos entorpece o de conectividad, que también más de alguno ha tenido problemas de conexión, y eso quizás lo desespera. (Docente 4)

El año pasado también llevamos a cabo un estudio de clase con el equipo y este año comenzamos con comunidades de aprendizaje, donde los coordinadores son como los facilitadores de este proceso de la reunión. (Integrante PIE 4)

En este período remoto, yo he sentido que ahi falta mucha comunicación. Falta, tenemos reuniones de ciclos, sí, pero son muy cortas ... Ahora es súper difícil porque no están los espacios ... no es la misma fluidez en el tema de comunicación y de ayuda. (Docente 1)

En este escenario, el colegio ha optado por ocupar el e-mail como mecanismo oficial de comunicación, que afecta y complejiza el trabajo colaborativo, pues a veces crea conflictos en la interpretación del mensaje.

\subsection{Prácticas colaborativas para la gestión dentro del establecimiento}

La gestión escolar es otra práctica colaborativa que se desarrolla durante la pandemia. Destaca la disposición a solucionar diversas problemáticas de directivos, asistentes de la educación e integrantes del equipo PIE. Este último contribuyó en la sistematización y análisis de información para retroalimentar el proceso de toma de decisiones de los equipos directivos, funcionando como puente en la comunidad:

[Los integrantes del PIE] Hacemos reuniones mensuales, veo el tema de los horarios, dificultades que vayan saliendo con estudiantes. Traspasamos a Unidad Técnica Pedagógica ... vamos haciendo como toda la bajada de información al equipo [técnico pedagógico] y a la misma vez la subida de las necesidades que van teniendo cada uno de los profesores. (Integrante PIE 3) 


\subsection{Roles de los miembros del establecimiento}

En esta dimensión, los participantes reconocen que todos los profesionales del establecimiento tienen un nivel de competencia muy alto, aunque distintos roles. Por ejemplo, el equipo directivo cumple un rol de supervisión y orientación. Esto implica un rol consultivo por parte de docentes y miembros del PIE sobre el estado de implementación de acciones exigidas:

Claro, porque en el fondo uno, uno nunca va a tener la autonomía total de la disciplina que enseña, o sea, siempre uno tiene que estar respondiendo a los superiores ... Entonces, para ello, para eso en el fondo uno recurre a los directivos. (Docente 4)

Los profesionales del establecimiento consideran que docentes e integrantes del equipo PIE con los que interactúan poseen un alto nivel de preparación y bastante dominio curricular, aunque con matices. Algunos entrevistados observan que algunas relaciones de colaboración se ven limitadas por formas de trabajo que dificultan la colaboración conjunta:

También son competitivos. Creo que a veces fallamos un poco, algunos más que otros, en la obsesión por el perfeccionar... antes de la pandemia éramos muy poco flexibles ... hemos aprendido con, como con harta dificultad, aprender a hacer un poco más moldeable. (Directivo 3)

Sin embargo, esto se balancea con el compromiso de enseñar en un ámbito social y contextual complejo, que implica adaptar procesos a las realidades de los estudiantes y recursos disponibles. Los roles, entonces, responden a la cultura institucional de la escuela, que permite una relación más estrecha y cercana entre los miembros de la comunidad escolar, incluyendo a profesionales, estudiantes y apoderados:

\footnotetext{
Las profesoras están pendientes de que, porque está [el estudiante], que no vino, todo, la emoción, porque está triste ... es más, más que un colegio tiene ese rol también como de una familia. Todos somos conocidos, todos se conocen ... Entonces yo creo que ... todos están pendientes del objetivo general, que es que todos aprendan. (Integrante PIE 1)
}

Los miembros del establecimiento destacan al equipo PIE, recalcando que el desarrollo profesional ciertamente influye en cómo se configuran los aspectos colaborativos de la escuela. Así, integrantes del PIE tomaron distintos roles durante la realización del trabajo a distancia en pandemia. Estos incluyeron el desarrollo de estrategias de enseñanza centradas en los estudiantes para garantizar el aprendizaje y evitar su deserción, adaptándose a sus características para garantizar que todos puedan acceder a las mismas oportunidades de aprendizaje:

Yo me acuerdo hace bastantes años atrás, cuando ingresamos era un trabajo bastante aislado ... ahora ya no los pueden, no los pueden retirar de la sala, a no ser que sea por cosas muy específicas, como alguna evaluación muy, muy especial. Por lo tanto, la participación es directa. (Docente 1)

El monitoreo constante del equipo PIE hacia las disciplinas facilitó lo anterior, junto con la cantidad de docentes y tiempo de trabajo directo con estudiantes y con apoderados, como parte del esfuerzo por asegurar el aprendizaje de estudiantes, el cual se vio expuesto debido al trabajo a distancia y los problemas de conexión, junto con la dificultad en la motivación de los estudiantes para participar activamente del proceso de educativo:

Yo me acuerdo de varias veces estar haciendo video, videollamada con la apoderada, para mostrarle como era, para que ella pudiera explicarle después a su estudiante. Entonces hubo cambio de roles, sí, hubo cambio de funciones también, nosotros no tan solo ya estábamos destinada al lenguaje y la matemática, no solo estábamos ya 
trabajando colaborativamente con el profesor de inglés ... música ... educación física ... teníamos contacto más directo con nuestro estudiante y pasamos a tener más cercanía y confianza. (Integrante PIE 3)

Además de lo anterior, el equipo PIE, en conjunto con los docentes del establecimiento, generó diversas estrategias de acompañamiento y contención emocional de los estudiantes, junto con la creación de planes de trabajo formativos acordes a la realidad emocional del estudiantado y sus familias, para evitar su deserción:

Yo creo que toda esta necesidad, que nació, fue también un objetivo que nosotros nos colocamos ... no porque nos hayan pedido los directivos [o] los docentes ... porque leímos que el Ministerio también daba tres indicadores que habia que priorizar y nosotros nos enfocamos por priorizar lo emocional en nuestro estudiante, evitando la deserción ... eso hizo que nosotros podamos cambiar estos roles y funciones. (Integrante PIE 1)

Sin embargo, esto no es compartido por todos los miembros del establecimiento. Si bien destacan y valoran su rol, algunos mencionan que el equipo PIE no siempre logra abarcar la totalidad de los espacios curriculares:

Entonces, en ese sentido la función del PIE se cumple, se cumple en el establecimiento. Pero lo que no se cumple o lo que faltaría como para poder mejorar, es que fuese quizás más completo en el sentido de poder abarcar todas las asignaturas y no solamente las que tengan su foco, digamos descendidos, cierto, donde, donde se pueden mejorar. (Docente 2)

\subsection{Desafios, beneficios y oportunidades del trabajo colaborativo en pandemia}

Para los directivos y equipo PIE, uno de los beneficios de la colaboración en pandemia fue la valorización del trabajo de los últimos, debido a su visibilización como pares profesionales, así como la promoción de estrategias de aprendizaje desde la co-docencia y el desarrollo profesional permanente. A futuro, esto generaría una oportunidad para pasar de un rol asistencial a uno más activo en los procesos de enseñanza de los estudiantes:

\footnotetext{
[Ahora] yo voy y como que yo intervengo y me siento como empoderada, y yo digo esto y le aporto, entonces me siento como que no tengo que tener un guión curricular ... en ese sentido que se nos abrió esa posibilidad, como esa puerta. Con el trabajo colaborativo. (Integrante PIE 1)

[Los integrantes del PIE] se transformaron en indispensables. Ahora es impensado que no haya una educadora [del PIE] colaborando ... en la clase. Los profesores, inclusive las piden ... porque se hicieron indispensables de verdad, que todos las valoraran. (Directivo 2)
}

La necesidad de afrontar nuevos desafíos consolidó nuevas estrategias de colaboración que permitieron motivar y llegar a los estudiantes durante el proceso de enseñanzaaprendizaje. Sin embargo, varios participantes muestran que aún es necesario expandir la co-docencia y ampliar el trabajo de los integrantes del programa PIE a todos los estudiantes del establecimiento y no solo los que están al alero del programa:

Entre el docente de aula y el docente de PIE ... la co-enseñanza existe, de hecho ... lo ideal es que esta co-enseñanza que hay en el establecimiento, este trabajo colaborativo entre el docente de aula y el equipo PIE, debe como expandir fronteras ... Entonces ahi yo creo que habría que darle como una vueltecita al objetivo del PIE, que en el fondo es apoyar el aprendizaje de todos los estudiantes, más que esos ... que tienen que atender. (Docente 4)

Si bien se menciona el manejo de plataformas digitales y el conocimiento sobre estrategias de enseñanza no-presenciales como una oportunidad de desarrollo profesional, la falta de conocimiento sobre estas obligó a los participantes a destinar tiempo extra para 
autoformarse sobre tecnologías educativas y poder ejercer su trabajo. Junto con esto, fue necesario generar nuevas estrategias de enseñanza que mantengan la motivación y atención de los estudiantes durante el trabajo a distancia para garantizar el derecho a la educación:

Por ejemplo, para mí, por lo menos, uno de los desafios fue ocupar las nuevas plataformas. Yo creo que eso fue un desafio para todos. Todas las plataformas digitales que nosotros no estábamos acostumbrados a ocuparlas. Eso para mí, puntualmente, fue como un nuevo desafio, todo lo que se relaciona a la tecnología y a la virtualidad. (Integrante PIE 3)

[El] desafío fue tratar de mantener a los niños activos, vigente, en el plan remoto durante todo el año. To creo que eso fue un gran desafío. Tratar de que siempre estuvieran presente en él, en su aprendizaje. (Docente 2)

A pesar de estas dificultades, los participantes destacan la creación de espacios para la priorización del trabajo emocional. En primer lugar, esto se expresó a través del trabajo colaborativo con apoderados, quienes se hacen partícipes en la enseñanza de sus hijos y debieron ser apoyados por profesionales del establecimiento en la adquisición de conocimientos disciplinares. En segundo lugar, la generación de estos vínculos también recae en las características contextuales del trabajo a distancia. En otras palabras, la escuela entra a la casa:

Fue una oportunidad de poder acercarnos más a nuestros estudiantes, a conocer su realidad que capaz antes no las conocíamos, porque abrimos las puertas de nuestros hogares. Tanto ellos conocernos a nosotros, conocimos a las mascotas casi de todos... no había quien no conociera a mi hija y su mascota, que ella misma la presentaba en clase. Entonces, fue una oportunidad de crear lazos con los estudiantes y crear lazos con los apoderados. (Integrante PIE 1)

Finalmente, la falta de conocimientos, recursos materiales, de conectividad, tiempo y tareas nuevas a realizar complejizó y precarizó altamente la labor de docentes e integrantes del PIE, afectando su vida privada. Esto se debe a que las nuevas exigencias, la sobrecarga laboral y las dinámicas de apoderados y estudiantes alteraron los horarios de trabajo y borraron la distinción clara entre dónde termina la vida laboral y dónde empieza la personal:

A lo mejor son malas prácticas, porque igual a veces nos salimos de horario, porque
lamentablemente nosotros ahora con la pandemia, nuestro horario es prácticamente
$24 / 7$. Porque a mi en sábado, domingo, los alumnos a veces o los papás, porque no
tuvieron tiempo en la semana, no pude comunicarme con ellos. Entonces igual uno
dice, me hubiese pasado a mí a lo mejor también trabajando y uno le contesta ... los
horarios traspasan. (Integrante del PIE 2)

Como parte de su diagnóstico, varios participantes mencionan que la normativa actual y los requerimientos ministeriales no garantizan el tiempo de trabajo que corresponde. Esto afecta a docentes, directivos e integrantes del PIE a nivel personal y a nivel laboral, incluyendo la organización del trabajo y participación en instancias necesarias para el desarrollo de prácticas colaborativas, entre otras. En el caso del equipo del PIE, su trabajo muchas veces sobrepasa lo establecido por la normativa. Para los docentes, la discordancia de los requerimientos del Servicio Local de Educación Pública (SLEP; institución sostenedora) hacia ellos y los directivos del establecimiento dificulta aspectos de la colaboración:

$\Upsilon$ a veces tenemos profesionales que tienen 3-4 cursos ... faltan horas para que se logre mayor efectividad y si se logra es porque aqui todos son comprometidos, y están casi hasta afuera de horario ... yo siento que eso es importante que alguna vez alguien 
estudió y se discuta, capaz, en los creadores y formadores de leyes ... la labor del profesor diferencial. (Integrante PIE 3)

[L]a organización pública de la educación, que ha sido muy variable, muy inconstante y también poco respetuosa de lo que ellos mismos establecen ... ha habido que estar estudiando sobre la marcha, algo que debió haberse preparado mejor para dar un funcionamiento más óptimo ... eso influye también en la colaboración directa del equipo directivo ... cuatro funcionarios distintos del Servicio Local le piden la misma información a la directora. (Docente 1)

Internamente, el desarrollo de la pandemia y su impacto en la labor de los docentes e integrantes del PIE permitió a los directivos identificar una serie de proyecciones para apoyar el trabajo y prácticas de colaboración. Así, mencionan una posible reconfiguración de los tiempos del trabajo profesional para evitar conflictos producto del cansancio:

Tiene que ver con un tema de evitar más conflictos, porque yo entiendo que la gente está súper reventada con esta cuestión de la pandemia, el encierro ... Es agotador para nosotros como líderes. Yo encuentro que es súper agotador, que es desgastante. (Directivo 3)

Finalmente, y a pesar de todas las dificultades mencionadas, los entrevistados reconocen que la pandemia los llevó a concretar prácticas colaborativas que no se habían podido desarrollar. Sin embargo, esto nace de la necesidad por hacer frente a una situación que afectó a los miembros del establecimiento a nivel laboral y personal:

Bueno, yo vivo la colaboración día a día porque tengo colegas que llegan y los busco de alguna u otra forma para que me ayuden cuando estoy muy ahogado, pero como escuela, como institución, no la habíamos vivido realmente. Era solamente una teoría y el estar en esta, en esta realidad, en este río que te estoy mencionando, tuvimos que hacer la balsa, tuvimos que buscar el... el que sabía amarrar, el que cortaba los palos y el que sabía navegar. Tuvimos que ponernos para poder navegar en él, en esta, no digo irrealidad, sino en esta realidad obligada que estábamos. (Directivo 4)

\section{Discusión y conclusiones}

El presente estudio entrega diversos hallazgos relacionados con el desarrollo profesional docente asociado al contexto colaborativo de una escuela bajo un escenario de emergencia sanitaria inédito en Chile y el mundo, los cuales se pueden desglosar desde un nivel macro, pasando con niveles intermedio y hasta el nivel micro propio de la escuela analizada.

En cuanto al nivel macro, se detecta una crítica al accionar de una reciente política pública educativa en Chile, específicamente al accionar de los SLEP (Raczynski et al., 2019), quienes abruman con labores extras en un contexto de pandemia que de por sí ya es demandante. Estos aspectos de los SLEP fueron advertidos previamente (Bellei, 2018), y lo que pareciera contradictorio, son los propios SLEP los que tienen que fomentar la colaboración efectiva y asesoría entre pares en y entre las escuelas bajo su administración (Solar et al., 2017). La evidencia de este estudio indica que falta una mayor conexión de las demandas del SLEP con el contex to y potencial desarrollo profesional en sus escuelas.

Por otro lado, respecto al nivel intermedio, entendiendo este como el contexto de la escuela, se vislumbra que un escenario adverso condiciona los patrones de colaboración, y con ello, el desarrollo profesional de los actores de la escuela. Así, en esta escuela ha emergido con fuerza el rol del equipo PIE y la inclusión educativa, pues con la pandemia se han exacerbado las inequidades socioeducativas, que es uno de los focos del PIE (Rodríguez Rojas, 2012). También las familias tienen un papel clave, en particular con las condiciones de conectividad que los estudiantes requieren en una educación remota de 
emergencia (Bond, 2020; UNICEF, 2020), y lo que ocurre en casos como el estudiado, donde miembros de la escuela socorren esta y otras situaciones para que el proceso educativo siga su curso. Esto ha conllevado un aprendizaje de docentes y directivos, pues han emergido aspectos colaborativos en un formato y foco diferente, y que va más allá de lo que ocurre "dentro" de la escuela, pues estos límites anteriormente físicos se han vuelto difusos en una educación preponderantemente online. No obstante, surge a su vez la disyuntiva de que no es claro lo que ocurrirá post-pandemia, en particular qué aprendizajes y parte del desarrollo realizado se mantendrán o cambiarán. En este sentido, es de esperar que exista un acompañamiento efectivo en esta transición, que involucra la articulación de las autoridades y actores involucrados en la política educativa con las comunidades educativas en su conjunto, en particular en potenciar las habilidades socioemocionales que visualizan docentes y directivos escolares para estos escenarios (Egan et al., 202 1).

Por último, en el nivel micro, considerando a la escuela en sí misma, la preponderancia del equipo PIE ha conllevado la aparición concreta de la co-docencia. No obstante, pareciera que este aspecto ha tenido diversas aristas en la escuela analizada, pues se contraponen las visiones de directivos, docentes y miembros del propio equipo PIE, lo cual ha sido destacado en investigaciones previas (Rodríguez Rojas, 2012), aunque en este caso es más bien asociado a la pandemia. A esto se agrega el potencial que tienen las comunidades de aprendizaje (CAP) en la escuela, que conlleva la transformación positiva de los centros educativos y su desarrollo profesional (Rodríguez de Guzmán, 2012). Sin embargo, se requiere superar diferentes escollos para su avance concreto y efectivo, lo cual es una oportunidad que se complejiza en pandemia (Cisternas et al., 2020; Silva, 2020).

A nivel individual se visualiza un importante papel de las habilidades socioemocionales o personales de cada actor escolar, en particular aquellas relacionadas con lo social y comunicacional, pues en contextos complejos como la pandemia -y actuando en modalidad on-line permanentemente-, se tiende a desdibujar las caras y afectos que existían presencialmente. Esto puede producir conflictos innecesarios, sobre todo si decrece la asertividad o no se lograr transmitir ni interpretar los tonos de los mensajes en modalidad remota, sumado a la coyuntura por Covid-19 (Villafuerte et al., 2020), lo cual tiene implicancias para el desarrollo profesional de docentes, directivos y equipos de apoyo. En la misma línea están las actitudes y rasgos personales, pues no es esperable que todos cuenten con las condiciones necesarias (personales o contextuales) para responder a las demandas de la pandemia, por lo que aspectos como la resiliencia y empatía, entre otros, se han convertido en claves a la hora de trabajar colaborativamente en contextos de emergencia (Gajardo et al., 2021; Román et al., 2020).

Esto involucra una expectativa y estado de desarrollo más profundo que lo presente hasta ahora, por lo que las proyecciones post-pandemia son diversas y alentadoras, aunque sin mayores certezas ni claridad, lo cual merma un pertinente desarrollo profesional acorde a los aspectos colaborativos sondeados, que involucra diferentes dimensiones contextuales (Khanal et al., 2021).

De acuerdo con lo anterior, es necesario profundizar en aquellas condiciones y dimensiones que promueven la colaboración en las escuelas, así como su articulación con el desarrollo profesional contextualizado de docentes, directivos y equipos de apoyo, con un énfasis en las enseñanzas, aprendizajes y desafíos que ha involucrado la pandemia y el escenario posterior a esta (Sahlberg, 2020), así como mayor investigación situada que enfatice las experiencias y lecciones de manera pertinente para los actores en los diferentes 
niveles del sistema educativo, cuya evidencia es esencial para las futuras acciones postpandemia.

\section{Referencias}

Aparicio-Molina, C. y Sepúlveda-López, F. (2019). Trabajo colaborativo docente: Nuevas perspectivas para el desarrollo docente. Revista Internacional de Investigación en Ciencias Sociales, 15(2), 119-133. https://doi.org./10.1590/2175-35392019017926

Ávalos, B. (2011). Teacher professional development in Teaching and Teacher Education over ten years. Teaching E Teacher Education, 27(1), 10-20. https://doi.org/10.1016/j.tate.2010.08.007

Ávalos, B. (2013). La formación inicial docente. En UNESCO-OREALC (Coord.), Antecedentes y criterios para la elaboración de políticas docentes en América Latina y el Caribe (pp. 37-56). CEPPE y UNESCO.

Ávalos-Bevan, B. y Bascopé, M. (2017). Teacher informal collaboration for professional improvement: beliefs, contexts, and experience. Education Research International, 1-13. https://doi.org/10.1155/2017/1357180

Bellei, C. (2018). Nueva educación pública: Contexto, contenidos y perspectivas de la desmunicipalización. Universidad de Chile

Bolívar, A. y Bolívar, M (2013). Construir la capacidad de mejora escolar: Liderazgo distribuido en una comunidad profesional de aprendizaje.Educ@rnos,10,11-34.

Bond, M. (2020). Schools and emergency remote education during the Covid-19 pandemic: A living rapid systematic review. Asian Journal of Distance Education, 15(2), 191-247.

Cisternas, D., Franco, G., Rodríguez, D., Verdugo, R., Guerra, N., Delgado, V. y Aguilar, R. (2020). Diálogos en pandemia: Algo de lo que hemos aprendido en la formación de comunidades de aprendizaje. Revista de Innovación en Enseñanza de las Ciencias, 4(1), 23-40.

Cochran-Smith, M., Villegas, A. M., Abrams, L., Chavez-Moreno, L., Mills, T. y Stern, R. (2015). Critiquing teacher preparation research: An overview of the field. Journal of Teacher Education, 66(2), 109-121. https://doi.org/10.1177/0022487114558268

Coskie, T. L. y Place, N. A. (2008). The national board certification process as professional development: The potential for changed literacy practice. Teaching and Teacher Education, 24(7), 1893-1906. https://doi.org/10.1016/j.tate.2008.02.005

Creswell, J. y Plano Clark, V. (2017). Designing and conducting mixed methods research. Sage.

Egan, S. M., Pope, J., Moloney, M., Hoyne, C. y Beatty, C. (202 1). Missing early education and care during the pandemic: The socio-emotional impact of the Covid-19 crisis on young children. Early Childhood Education Journal, 49, 925-934. https://doi.org/10.1007/s10643-021-01193-2

Figueroa-Céspedes, I., Sepúlveda Guajardo, G., Soto Cárcamo, J. y Yáñez-Urbina, C. (2020). Coenseñanza entre docentes de educación general básica y educadoras diferenciales: incidentes críticos de la práctica colaborativa en proyectos de integración educativa. Pensamiento Educativo. Revista de Investigación Educacional Latinoamericana, 57(1), 1-15. https://doi.org/10.7764/PEL.57.1.2020.1

Gajardo-Asbún, K., Paz-Maldonado, E. y Salas, G. (2021). Conceptions of teacher trainers from Covid-19. A comparative study in three regions of Chile. Revista Española de Educación Comparada, 38, 69-89. https://doi.org/10.5944/REEC.38.2021.28867 
Gajardo, J., Salazar, M. y Maya, A. (2021). Enseñar historia en tiempos de pandemia: un estudio de caso en una escuela chilena. Revista Española de Educación Comparada, 38, 90-111. https://doi.org/10.5944/reec.38.2021.28996

García-Martínez, I., Montenegro-Rueda, M., Molina-Fernández, E. y Fernández-Batanero, J. M. (2021). Mapping teacher collaboration for school success. School Effectiveness \& School Improvement. https://doi.org/10.1080/09243453.2021.1925700

Guerra, P., Rodriguez, M. y Zañartu, C. (2020). Comunidades profesionales de aprendizaje en educación parvularia en Chile. Cadernos de Pesquisa, 50(177), 828-844. https://doi.org/10.1590/198053146858

Hairon, S. y Tan, C. (2017). Professional learning communities in Singapore and Shanghai: Implications for teacher collaboration. Compare: A Journal of Comparative and International Education, 47(1), 91-104. https://doi.org/10.1080/03057925.2016.1153408

Jonsen, K. y Jehn, K. A. (2009). Using triangulation to validate themes in qualitative studies. Qualitative Research in Organizations and Management: An International Journal, 4(2), 123-150. https://doi.org/10.1590/198053146858

Jurasaite-Harbison, E. y Rex, L. A. (2010). School cultures as contexts for informal teacher learning. Teaching and Teacher Education, 26(2), 267-277. https://doi.org/10.1016/j.tate.2009.03.012

Khanal, P., Bento, F. y Tagliabue, M. (2021). A scoping review of organizational responses to the covid-19 pandemic in schools: A complex systems perspective. Education Sciences, $11(115)$, 1-21. https://doi.org/10.3390/educsci1 1030115

Lizana, P., Vega-Fernadez, G., Gomez-Bruton, A., Leyton, B. y Lera, L. (2021). Impact of the Covid-19 pandemic on teacher quality of life: a longitudinal study from before and during the health crisis. International Journal of Environmental Research and Public Health, 18(7), 3764. https://doi.org/10.3390/ijerph 18073764

López, V., Julio, C., Pérez, M., Morales, M. y Rojas, C. (2014). Barreras culturales para la inclusión: Políticas y prácticas de integración en Chile. Revista de Educación, 363, 256-281. https://doi.org/10-4438/1988-592X-RE-2012-363-180

MINEDUC. (2009). Decreto $170 . \quad$ https://especial.mineduc.cl/wpcontent/uploads/sites/31/2018/06/DTO-170_21-ABR-2010.pdf

MINEDUC. (2017). Orientaciones sobre estrategias diversificadas de enseñanza para educación básica, en el marco del decreto 83/2015. https://especial.mineduc.cl/wpcontent/uploads/sites/31/2017/05/ORIENTACIONES_D83_Web_05-2017.pdf

MINEDUC (2019). Trabajo colaborativo y desarrollo profesional docente en la escuela. https://www.cpeip.cl/wp-content/uploads/2019/03/trabajo-colaborativo_marzo2019.pdf

Moolenaar, N. M. (2012). A social network perspective on teacher collaboration in schools: Theory, methodology, and applications. American Journal of Education, 199(1), 7-29. https://doi.org /10.1086/667715

Muñoz, M., López, M. y Assaél, J. (2015). Concepciones docentes para responder a la diversidad: ¿Barreras o recursos para la inclusión educativa? Psicoperspectivas, 14(3), 68-79. https://doi.org/10.5027/psicoperspectivas-Vol14-Issue3-fulltext-646

Navarro-Aburto, B., Arriagada-Puschel, I., Osse-Bustingorry, S. y Burgos-Videla, C. (2016). Adaptaciones curriculares: Convergencias y divergencias de su implementación en el profesorado chileno. Revista Electrónica Educare, 20(1), 1-18.

https://doi.org/10.15359/ree.20-1.1 
Peel, K. (2020). A beginner's guide to applied educational research using thematic analysis. Practical Assessment, Research, and Evaluation, 25(1), 1-15. https://doi.org/10.7275/ryr5-k983

Philpott, C. y Oates, C. (2017). Professional learning communities as drivers of educational change: The case of learning rounds. Journal of Educational Change, 18(2), 209-234. https://doi.org/10.1007/s 10833-016-9278-4

Raczynski, D., Rivero, R. y Yáñez, T. (2019). Nivel intermedio del sistema escolar en Chile: normativa y visión de los sostenedores acerca de las funciones, preparación para el cargo y las prácticas. Calidad en la Educación, (51), 382-420.

Ramos-Huenteo, V., García-Vásquez, H., Olea-González, C., Lobos-Peña, K. y Sáez-Delgado, F. (2020). Percepción docente respecto al trabajo pedagógico durante la Covid-19. CienciAméRica, 9(2), 334-353. https://doi.org/10.33210/ca.v9i2.325

Rodríguez de Guzmán, J. (2012). Comunidades de aprendizaje y formación del profesorado. Tendencias Pedagógicas, 19, 67-86

Rodríguez-Gómez, D. y Gairín-Sallán, J. (2015). Innovación, aprendizaje organizativo y gestión del conocimiento en las instituciones educativas. Educación, 24(46), 73-90.

Rodríguez Rojas, F. F. (2012a). La percepción del trabajo colaborativo en la gestión curricular de profesores de educación regular y educación especial en Programas de Integración Escolar de la comuna de Tomé [Tesis de Magíster]. Universidad del Bio-bio.

Rodríguez Rojas, F. F. y Ossa, C. (2014). Valoración del trabajo colaborativo entre profesores de escuelas básicas de Tomé, Chile. Estudios Pedagógicos, 4O(2), 303-319. https://doi.org/10.4067/S0718-07052014000300018

Román, F., Forés, A., Calandri, I., Gautreaux, R., Antúnez, A., Ordehi, D., ... y Allegri, R. (2020). Resiliencia de docentes en distanciamiento social preventivo obligatorio durante la pandemia de Covid-19. Journal of Neuroeducation, 1(1), 76-87.

https://doi.org/10.1344/joned.v1i1.31727

Sahlberg, P. (2020). Will the pandemic change schools?. Journal of Professional Capital and Community, 5(3/4), 359-365. https://doi.org/10.1108/JPCC-05-2020-0026

Salas, G., Santander, P., Precht, A., Scholten, H., Moretti, R. y López-López, W. (2020). Covid-19: impacto psicosocial en la escuela en Chile. Desigualdades y desafíos para Latinoamérica. Avances en Psicología Latinoamericana, 38(2), 1-17.

https://doi.org/10.12804//revistas.urosario.edu.co/apl/a.9404

Sato, K. y Kleinsasser, R. C. (2004). Beliefs, practices, and interactions of teachers in a Japanese high school English department. Teaching and Teacher Education, 20(8), 797-816. https://doi.org/10.1016/j.tate.2004.09.004

Scott, J. (2000). Social network analysis: A handbook. Sage.

Silva, H. (2020). Trayectoria de comunidades profesionales de aprendizaje: Desafíos y oportunidades en pandemia. Revista de Innovación en Enseñanza de las Ciencias, 4(1), 143-160. https://doi.org/10.5027/reinnec.V4.I1.88

Skerrett, A. (2010). “There's going to be community. There's going to be knowledge”: Designs for learning in a standardised age. Teaching and Teacher Education, 26(3), 648-655. https://doi.org/10.1016/j.tate.2009.09.017

Solar, H., Treviño, E., San Martín, E. y Ayala, P. (2017). Modelo de apoyo para el desarrollo profesional docente para estructuras de gobierno municipal y Servicios Locales de 
Educación (SLE). En I. Irarrázaval, E. Piña, M. Letelier, M. e I. Jeldes (Eds.), Propuestas para Chile Concurso Políticas Públicas 2017 (pp. 75-104). Centro de Políticas Públicas UC.

Stewart, D. y Shamdasani, P. (2014). Focus groups: Theory and practice. Sage.

Tenorio, S. (2005). La integración escolar en Chile: perspectiva de los docentes sobre su implementación. REICE. Revista Iberoamericana sobre Calidad, Eficacia y Cambio en Educación, 3(1), 823-831.

UNESCO-OREALC. (2013). Temas críticos para formular nuevas políticas docentes en América Latina y el Caribe: el debate actual. CEPPE y UNESCO.

UNICEF. (2020). How many children and young people have internet access at home? Estimating digital connectivity during the Covid-19 pandemic. UNICEF.

Urbina, C., Basualto, P., Durán, C. y Miranda, P. (2017). Prácticas de co-docencia: el caso de una dupla en el marco del Programa de Integración Escolar en Chile. Estudios Pedagógicos, 43(2), 355-374. https://doi.org/10.4067/So718-07052017000200019

Vaillant, D. y Marcelo C. (2015). El A, B, C, D de la formación docente. Narcea.

Vaillant, D. (2016). Trabajo colaborativo y nuevos escenarios para el desarrollo profesional docente. Revista Hacia un Movimiento Pedagógico Nacional, 60, 7-13.

Vangrieken, K., Dochy, F., Raes, E. y Kyndt, E. (2015). Teacher collaboration: A systematic review. Educational research review, 15, 17-40. https://doi.org/10.1016/j.edurev.2015.04.002

Vangrieken, K., Grosemans, I., Dochy, F. y Kyndt, E. (2017). Teacher autonomy and collaboration: A paradox? Conceptualising and measuring teachers' autonomy and collaborative attitude. Teaching and Teacher Education, 67, 302-315. https://doi.org/10.1016/j.tate.2016.10.001

Venegas, P., Campos, J., Cortés, C. y Novoa, X. (2010). Comunidades de aprendizaje: una estrategia de participación y desarrollo profesional docente en Chile. Buenos Aires: Foro Latinoamericano de Políticas Educativas.

Villafuerte, J., Cevallos, Y. y Vidal, J. (2020). Rol de los docentes ante la crisis del Covid-19, una mirada desde el enfoque humano. REFCalE: Revista Electrónica Formación y Calidad Educativa, 8(1), 134-150.

Wasserman, S. y Faust, K. (1994). Social network analysis: methods and applications. Cambridge University Press.

Yin, R. (2003). Case study research: design and methods. Sage.

\section{Breve CV de los/as autores/as}

\section{Juan Pablo Queupil Quilamán}

Doctor en Educación por la Universidad Estatal de Pennsylvania, Estados Unidos. Magíster en Gestión y Dirección de Empresas, Ingeniero Civil Industrial y Licenciado en Ciencias de la Ingeniería por la Universidad de Chile. Director del Centro de Investigación para la Transformación SocioEducativa (CITSE), y académico e investigador de la Universidad Católica Silva Henríquez (UCSH), Chile. Es profesor del Magíster en Educación de la UCSH e integrante del grupo de investigación de "liderazgo y colaboración para la mejora educativa" en la misma. Miembro de la Red de Investigadores en Educación Chilena (RIECH). Desarrolla su actividad investigadora centrada en la gestión y liderazgo educacional, colaboración en educación, inclusión y justicia educacional, y política educativa. Email: jqueupil@ucsh.cl 
ORCID ID: http://orcid.org/0000-0002-7324-9275

\section{Catalina Cuenca Vivanco}

Licenciada en Lengua y Literatura Inglesas de la Universidad de Chile y Máster en Ciencias de la Educación con mención en Lectura, Escritura y Literacidad de la Universidad de Pensilvania, Estados Unidos. Actualmente trabaja como asistente de investigación y formación en la Universidad Católica Silva Henríquez y la Universidad Metropolitana de Ciencias de la Educación (Chile). Entre sus intereses de investigación se encuentran la formación inicial docente para la justicia social, indagación docente, literalidad crítica, literacidad académica y análisis de políticas educativas. Ha trabajado en proyectos de investigación sobre análisis de política educativa para la formación inicial docente y su implementación, ideologías curriculares de profesores en formación, desarrollo de prácticas pedagógicas innovadoras de profesores en formación y de aula, y desarrollo de prácticas colaborativas entre equipos docentes y directivos en establecimientos educativos públicos y privados. Email: catalinapcv@gmail.com

ORCID ID: https://orcid.org/0000-0003-3914-0548

\section{César Maldonado Díaz}

Candidato a Doctor en Educación por la Universidad Alberto Hurtado y Universidad Diego Portales. Magíster en Educación (Universidad de Chile) y Profesor de Historia, Geografía y Cs. Sociales (PUCV). Académico e investigador en el Centro de Investigación para la Transformación SocioEducativa (CITSE) de la Universidad Católica Silva Henríquez. Se ha especializado en las siguientes líneas de investigación: el estudio de las prácticas de co-enseñanza entre docentes con especialidades diversas en aulas escolares chilenas. Además, le convoca el estudio de la colaboración docente, el rol de los saberes pedagógicos en el desarrollo de la formación inicial y como otros actores intervienen en la formación de docentes. Lo anterior, lo hace desde un enfoque sociológico bernsteniano propio del estudio del discurso pedagógico puesto en acto. Email: cmaldonadod@ucsh.cl

ORCID ID: https://orcid.org/0000-0001-6507-8093 